\title{
Carboxymethyl-chitosan attenuates inducible nitric oxide synthase and promotes interleukin-10 production in rat chondrocytes
}

\author{
YING KONG, YUANMIN ZHANG, XIAOWEI ZHAO, GUODONG WANG and QINGKUAN LIU
}

Department of Bone and Joint Surgery, Affiliated Hospital of Jining Medical University, Jining, Shandong 272029, P.R. China

Received March 30, 2017; Accepted August 10, 2017

DOI: $10.3892 /$ etm.2017.5258

\begin{abstract}
Osteoarthritis (OA) is a common age-related degenerative joint disease, which is caused by the breakdown of joint cartilage and the underlying bone. Carboxymethyl (CM)-chitosan is a soluble derivative of chitosan that has similar physicochemical properties to the extracellular proteoglycans identified in hyaline cartilage. Previous studies have demonstrated that CM-chitosan serves a protective role in a rabbit OA model. The aim of the present study was to investigate the effect of CM-chitosan on NO production and inflammation through its upregulation of interleukin (IL)-10, and the activation of the janus kinase (JAK)/signal transducer and activator of transcription (STAT)/suppressor of cytokine signaling (SOCS) signaling pathway. In the present study primary rat chondrocytes were induced to inflammation with $2 \mu \mathrm{g} / \mathrm{ml}$ lipopolysaccharide. The cells were subsequently subjected to increasing concentrations of CM-chitosan $(50,100$ and $200 \mu \mathrm{g} / \mathrm{ml})$ and the relative mRNA and protein expression of inducible nitric oxide synthase (iNOS), IL-10, JAK1, STAT3 and SOCS3 were measured by RT-qPCR and western blot analysis respectively. The results revealed that $\mathrm{CM}$-chitosan attenuated inflammation by significantly reducing iNOS expression and upregulating the anti-inflammatory cytokine IL-10 in a dose-dependent manner $(\mathrm{P}<0.05)$. The expression of JAK1, STAT3 and SOCS3 were also significantly upregulated by $\mathrm{CM}$-chitosan (all $\mathrm{P}<0.05$ ). The protective role of CM-chitosan against NO production was due to its upregulation of IL-10 and its activation of the JAK/STAT/SOCS signaling pathway.
\end{abstract}

\section{Introduction}

Osteoarthritis (OA) is a common age-related degenerative joint disease, which is caused by the breakdown of joint cartilage

Correspondence to: Dr Qingkuan Liu, Department of Bone and Joint Surgery, Affiliated Hospital of Jining Medical University, 89 Gu'huai Road, Jining, Shandong 272029, P.R. China

E-mail: liu86829234@hotmail.com

Key words: carboxymethyl-chitosan, osteoarthritis, inflammation, nitric oxide, interleukin-10 and the underlying bone (1). OA is a major cause of severe joint pain, knee swelling and physical disability in elderly patients (2). A previous study reporting an epidemiological survey revealed that $>10 \%$ of adults $>60$ years old suffer from $\mathrm{OA}$, and that the incidence rate increases with age and body mass index (3). An understanding of the underlying mechanisms of pathogenesis in $\mathrm{OA}$ is imperative to the development of novel treatments against the disease.

Cartilage is an important component of joints and is comprised of highly specialized chondrocyte cells (4). The principal function of chondrocytes is to produce the extracellular matrix, which includes collagen and proteoglycan, so as to maintain the integrity and stability of the joint (4). $\mathrm{OA}$ is characterized by the extensive loss of proteoglycan content surrounding the chondrocytes (5). Several previous studies have demonstrated that NO is a key mediator in the pathogenesis of OA (6-8). Nitrite has been identified as being increased in the synovial fluid and serum of patients with OA, which suggests an increased rate of NO synthesis (9). In experimentally induced models of OA, NO production was induced by the proinflammatory cytokine interleukin (IL)-1 $\beta$ and was associated with chondrocyte apoptosis (10). NO was revealed as promoting lipocalin-2 production and decreasing chondrocyte vitality (11). Endoplasmic reticulum stress also contributed to NO production and chondrocyte apoptosis (12).

For patients with early or middle stage OA, a non-arthroplasty treatment regime is typically prescribed, which is focused on the control of symptoms including the imbalance of cartilage homeostasis (13). Previous studies have demonstrated that chitosan and its derivatives may serve a protective role in $\mathrm{OA}(14,15)$. Chitosan is a linear polysaccharide and carboxymethyl (CM)-chitosan is a soluble derivative of chitosan. Chitinous materials have been described in many previous studies as having antioxidant, anti-inflammatory, antibacterial, anticancer and antifungal properties (16-18). Chitinous materials have similar physiochemical properties to the extracellular proteoglycans located in hyaline cartilage, and they have been used in animal models to prevent OA (19). CM-chitosan may inhibit matrix metalloproteinase (MMP) expression and protect rabbit chondrocytes from IL-1 $\beta$ induced apoptosis $(20,21)$.

In the present study it has been hypothesized that the protective role of CM-chitosan may be associated with $\mathrm{NO}$ production, and the role of CM-chitosan in inducible NO synthase (iNOS) expression has been investigated. The janus 
kinase (JAK)/signal transducer and activator of transcription (STAT)/suppressor of cytokine signaling (SOCS) signaling pathway has been revealed to be associated with iNOS expression $(22,23)$; therefore, it was also investigated whether $\mathrm{CM}$-chitosan was able to attenuate an inflammatory reaction by activating the JAK/STAT/SOCS signaling pathway.

\section{Materials and methods}

Isolation of chondrocytes. A total of 8 four-week old male Sprague-Dawley rats (180-200 g; Shandong Lukang Record Pharmaceutical Co., Ltd., Jining, China) were used for the isolation of articular chondrocytes as previously reported (24). All rats were allowed to eat and drink freely and housed at $22-25^{\circ} \mathrm{C}$ with $45-75 \%$ relative humidity and a $12 \mathrm{~h}$ light/dark cycle. Briefly, cartilage tissue from the knee joint was rinsed with PBS containing $100 \mu \mathrm{g} / \mathrm{ml}$ streptomycin and $100 \mathrm{U} / \mathrm{ml}$ penicillin. Chondrocytes were released from cartilage tissue by $0.2 \%$ type II collagenase digestion at $37^{\circ} \mathrm{C}$ for $6 \mathrm{~h}$ and filtered with a 150 mesh strainer. All cells were cultured in Dulbecco's Modified Eagle's medium (DMEM; Gibco; Thermo Fisher Scientific, Inc., Waltham, MA, USA) supplemented with $10 \%$ fetal bovine serum (Gibco; Thermo Fisher Scientific, Inc.) in a humidified hood with $5 \% \mathrm{CO}_{2}$ at $37^{\circ} \mathrm{C}$ for $24 \mathrm{~h}$. Primary cell cultures were used in all experiments. The animal protocol used in the present study was approved by the Animal Use Committee of Jining Medical University (Jining, China) prior to the commencement of the study.

Treatment of cells. Isolated chondrocytes were plated at a density of $1.3 \times 10^{5}$ cells/well in 6-well plates and incubated for $24 \mathrm{~h}$ at $37^{\circ} \mathrm{C}$. Fresh DMEM with $2 \mu \mathrm{g} / \mathrm{ml}$ lipopolysaccharide (LPS) was added to treat the cells for $4 \mathrm{~h}$. Different concentrations $(0,50,100$ and $200 \mu \mathrm{g} / \mathrm{ml})$ of CM-chitosan (Hebei Qian Sheng Biotechnology Co., Ltd., Shijiazhuang, China) were then added at $37^{\circ} \mathrm{C}$ for $24 \mathrm{~h}$. Chondrocytes without LPS treatment were used as the negative control.

Quantification of $m R N A$ by reverse transcription-quantitative polymerase chain reaction $(R T-q P C R)$. Total RNA was extracted from the harvested chondrocytes using the RNAiso plus kit (Takara Biotechnology Co., Ltd., Dalian, China) according to the manufacturer's protocol. cDNA was synthesized using the PrimeScript RT Master Mix kit (Takara Biotechnology Co., Ltd.) according to the manufacturer's protocol in a $25-\mu 1$ reaction volume containing $1 \mu \mathrm{g}$ template RNA. Subsequently qPCR was performed using the SYBR premix EX Taq kit (Takara Biotechnology Co., Ltd.) according to the manufacturer's protocol. The amplification conditions were: Initial denaturation at $94^{\circ} \mathrm{C}$ for $2 \mathrm{~min}$ and 40 cycles of denaturation at $94^{\circ} \mathrm{C}$ for $30 \mathrm{sec}$, annealing at $56^{\circ} \mathrm{C}$ for $40 \mathrm{sec}$ and extension at $72^{\circ} \mathrm{C}$ for $30 \mathrm{sec}$. The endogenous control used in this study was GAPDH. The primer sequences used in the PCR amplification were as follows: iNOS, forward 5'-CCT TGTTCAGCTACGCCTTC-3' and reverse 5'-CATGGTGAA CACGTTCTTGG-3' (565 bp); IL-10, forward 5'-AATCTG TGTTGTTTAAGCTGTTTCC-3' and reverse 5'-TTTATT CAAAACGAGGATCTGCTAC-3' (466 bp); JAK1, forward 5'-TGTGGCTGCTGACAAGTGGAG-3' and reverse 5'-ATG ATGGCTCGGAAGAAAGGT-3' (215 bp); STAT3, forward
5'-ATGGGTTTCATCAGCAAGGAG-3' and reverse 5'-GGG AATGTCAGGGTAGAGGTAGAC-3' (279 bp); SOCS3, forward 5'-ACCAGCGCCACTTCTTCACA-3' and reverse 5'-GTGGAGCATCATACTGATCC-3' (450 bp); and GAPDH, forward 5'-GGCACAGTCAAGGCTGAGAATG-3' and reverse 5'-ATGGTGGTGAAGACGCCAGTA-3' (143 bp). To compare the mRNA expression levels of targeted genes, the relative expression ratio was calculated using the $2^{-\Delta \Delta \mathrm{Cq}}$ method, as previously reported (25) and standardized with the control group.

Determination of protein expression by western blotting. Total protein was extracted from the chondrocytes by lysing the cells in a radioimmunoprecipitation assay lysis buffer $(50 \mathrm{mM}$ Tris $\mathrm{pH} 7.4,150 \mathrm{nM} \mathrm{NaCl}, 1 \%$ nonyl phenoxypolyethoxylethanol-40, $0.5 \%$ sodium deoxycholate and protease inhibitor cocktail (Santa Cruz Biotechnology, Inc., Dallas, TX, USA). The protein concentration was determined via bicinchoninic acid assay. Protein samples (30 $\mu$ g each sample per lane) were separated by $10 \%$ SDS-PAGE. Following electrophoresis, the proteins were transferred to polyvinylidene difluoride membranes (EMD Millipore, Billerica, MA, USA) and blocked with $5 \%$ skimmed milk at $4^{\circ} \mathrm{C}$ overnight. The membranes were subsequently probed with the following mouse monoclonal primary antibodies against iNOS (1:500; sc-7271), IL-10 (1:1,000; sc-32815), JAK1 (1:1,000; sc-1677), STAT3 $(1: 1,000 ;$ sc-8019), SOCS3 (1:500; sc-73045) and GAPDH (1:1,000; sc-47724; all Santa Cruz Biotechnology, Inc.) at room temperature for $2 \mathrm{~h}$. The membranes were washed three times with TBST (10 mM Tris, $150 \mathrm{mM} \mathrm{NaCl,} \mathrm{0.05 \%} \mathrm{Tween-20)}$ and then incubated with horseradish peroxidase-conjugated secondary antibody against mouse immunoglobulin G (1:2,000; sc-516102; Santa Cruz Biotechnology, Inc.) at room temperature for $2 \mathrm{~h}$. Blots were visualized by the enhanced chemiluminescence method (P0018; Beyotime Institute of Biotechnology, Haimen, China). The bands were analyzed by Quantity One software v4.62 (Bio-Rad Laboratories, Inc., Hercules, CA, USA).

Statistical analysis. Each experiment was repeated in triplicate and representative results were presented. SPSS version 20.0 software (IBM Corp., Armonk, NY, USA) was used for data analysis. Data are presented as the mean \pm standard deviation. Comparison among groups was analyzed by one-way analysis of variance with Dunnett's post hoc test. $\mathrm{P}<0.05$ was considered to indicate a statistically significant difference.

\section{Results}

Treatment with CM-chitosan causes a reduction in iNOS production in a dose dependent manner. Elevated NO concentration is typically observed in patients with OA (7). $\mathrm{NO}$ is synthesized by the NO synthase family in vivo, of which iNOS is a member. Therefore, the effect of CM-chitosan on iNOS production in chondrocytes was investigated. As revealed in Fig. 1, LPS exposure significantly increased the expression of iNOS mRNA in chondrocytes compared with the control group. However, treatment with increasing concentrations of CM-chitosan caused inhibition of iNOS 
A

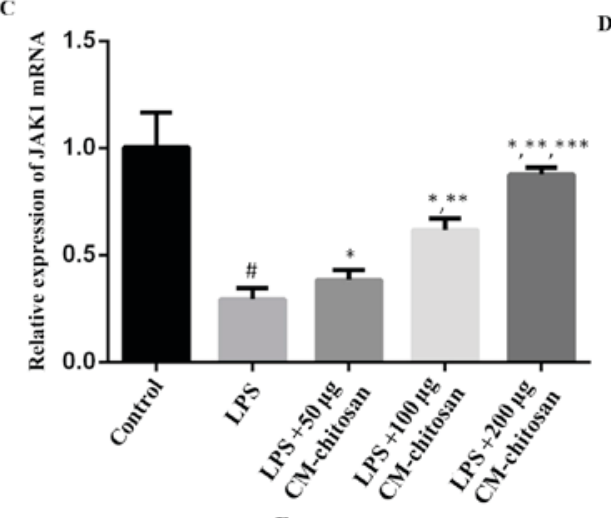

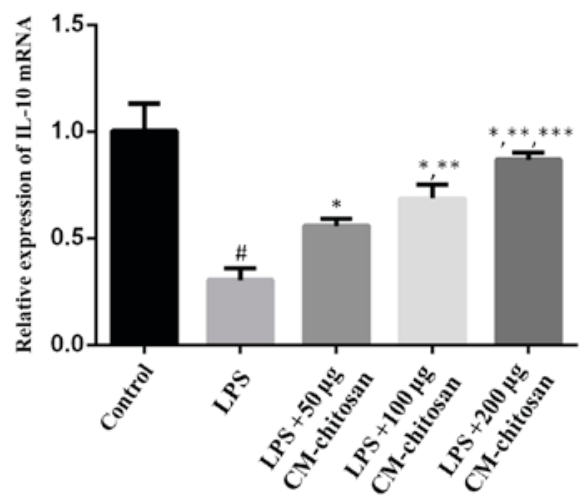

D

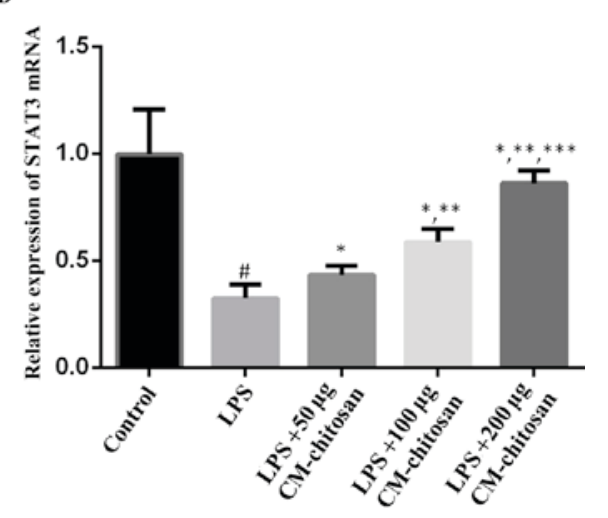

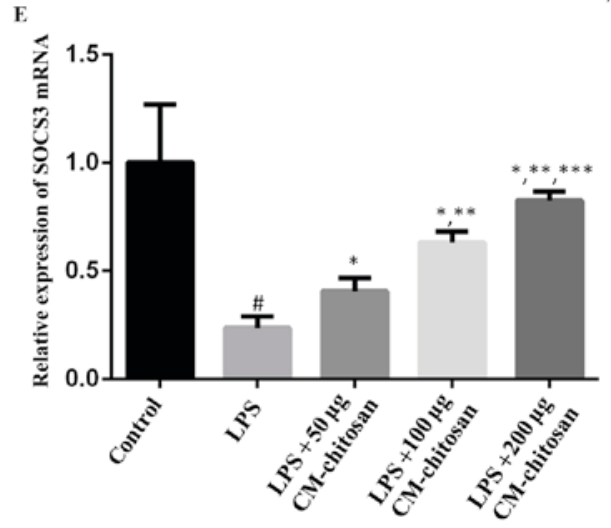

Figure 1. Effects of CM-chitosan on the mRNA levels of iNOS, IL-10, JAK1, STAT3 and SOCS3 in LPS-induced rat chondrocytes. Rat chondrocytes were treated with LPS and subsequently increasing concentrations (50, 100 and $200 \mu \mathrm{g} / \mathrm{ml})$ of CM-chitosan were added. Reverse transcription-quantitative polymerase chain reaction was used to measure the relative mRNA levels of (A) iNOS, (B) IL-10, (C) JAK1 (D) STAT3 and (E) SOCS3. Data are presented as the mean \pm standard deviation. ${ }^{\#} \mathrm{P}<0.05$ vs. the control group, ${ }^{*} \mathrm{P}<0.05$ vs. the LPS group, ${ }^{* *} \mathrm{P}<0.05$ vs. the LPS $+50 \mu \mathrm{g}$ CM-chitosan group, and ${ }^{* * * *} \mathrm{P}<0.05$ vs. the LPS+100 $\mu \mathrm{g}$ CM-chitosan group. CM, carboxymethyl; iNOS, inducible nitric oxide synthase; IL, interleukin; JAK, janus kinase; STAT, signal transducer and activator of transcription; SOCS suppressor of cytokine signaling; LPS, lipopolysaccharide.

mRNA in a dose-dependent manner. Treatment with 50, 100 and $200 \mu \mathrm{g} / \mathrm{ml} \mathrm{CM}$-chitosan caused a decline of iNOS mRNA at a fold rate of $2.79,2.08$, and 1.42 respectively, compared with the LPS group Fig. 2 demonstrates that the protein expression of iNOS was also decreased by CM-chitosan in a dose dependent manner.

Treatment with CM-chitosan increases IL-10 production in a dose dependent manner. IL-10 is well known as an anti-inflammatory cytokine. A previous study has revealed that iNOS was induced in IL-10-deficient mice (26). The effects of CM-chitosan on IL-10 production were analyzed in the present study. As demonstrated in Fig. 1B, IL-10 mRNA was downregulated by LPS exposure. Treatment with increasing concentrations of CM-chitosan was revealed to recover IL-10 production to near-control levels in a dose-dependent manner. There was no significant difference between the IL-10 mRNA level of the control group and the group treated with $200 \mu \mathrm{g} / \mathrm{ml}$ CM-chitosan. The level of IL-10 protein demonstrated the same trend and also increased in a dose dependent manner when treated with CM-chitosan (Fig. 2A and C). These results indicated that iNOS inhibition was associated with IL-10 induction.

CM-chitosan activates the JAK/STAT/SOCS signaling pathway. The present study also investigated whether the JAK/STAT/SOCS signaling pathway was activated by CM-chitosan. The mRNA levels of JAK1, STAT3 and 

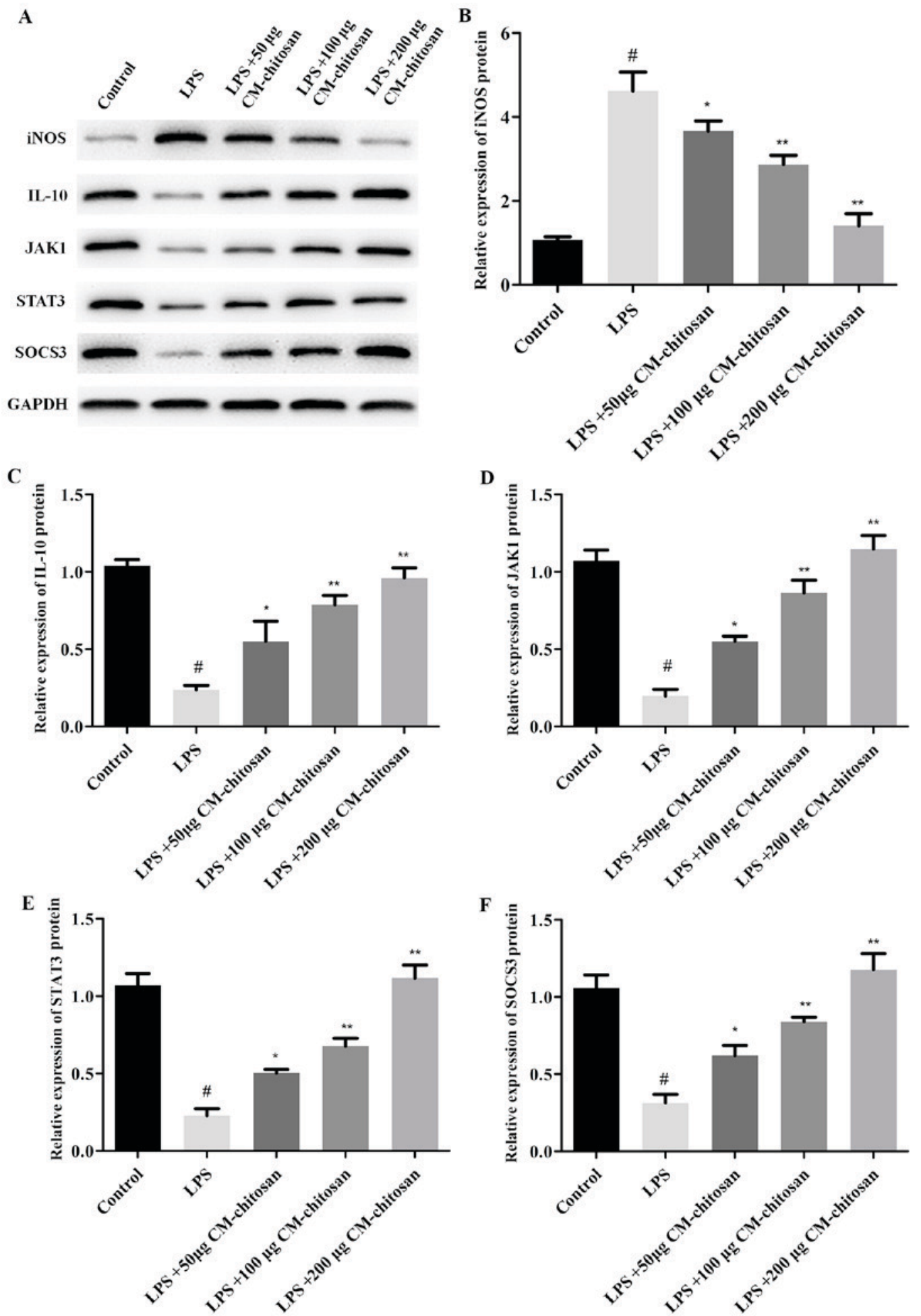

Figure 2. Effects of CM-chitosan on the protein levels of iNOS, IL-10, JAK1, STAT3 and SOCS3 in LPS-induced rat chondrocytes. Rat chondrocytes were treated with LPS and subsequently increasing concentrations (50,100 and $200 \mu \mathrm{g} / \mathrm{ml}$ ) of CM-chitosan were added. (A) A western blot analysis was performed to detect the proteins present in the rat chondrocytes. These results were quantified and the relative protein expression levels of (B) iNOS, (C) IL-10, (D) JAK1, (E) STAT3 and (F) SOCS3 were presented. Data are presented as the mean \pm standard deviation. ${ }^{~} \mathrm{P}<0.05$ vs. the control group, ${ }^{\text {"P }}<0.05$ vs. the LPS group and ${ }^{* *} \mathrm{P}<0.05$ vs. the LPS $+50 \mu \mathrm{g}$ CM-chitosan group. CM, carboxymethyl; iNOS, inducible nitric oxide synthase; IL, interleukin; JAK, janus kinase; STAT, signal transducer and activator of transcription; SOCS suppressor of cytokine signaling; LPS, lipopolysaccharide.

SOCS3 were all suppressed in chondrocytes exposed to LPS (Fig. 1D-F), and promoted by CM-chitosan treatment in a dose dependent manner. Similarly, western blot analysis demonstrated that the protein expression of JAK1, STAT3 and SOCS3 were also upregulated in CM-chitosan groups (Fig. 2).
When treated with $200 \mu \mathrm{g} / \mathrm{ml}$ of CM-chitosan all three proteins were expressed at a markedly higher level than the control. These results indicated that the JAK/STAT/SOCS signaling pathway was activated by CM-chitosan and this activation was associated with $\mathrm{NO}$ inhibition and IL-10 induction. 


\section{Discussion}

Loss of cartilage is one of the primary symptoms identified in patients with OA (27). Articular cartilage cannot be repaired if it is damaged as chondrocytes, which are the only cell type in cartilage, have a very low metabolic activity. This feature of chondrocytes makes the treatment of OA challenging (3). To the best of our knowledge there are currently no approved disease-modifying drugs against OA. Inflammation plays a central role in OA and Zheng et al (28) have recently reviewed the use of anti-cytokine approaches to improve the symptoms of $\mathrm{OA}$ as well as stabilizing the tissue structure. Inhibition of NO production is another potential strategy to treat OA as NO production is closely associated with inflammatory reactions (10). Chemical substances including echinocystic acid, selenomethionine and piperine have been identified in previous studies as inhibitors of iNOS production and therefore attenuators of inflammation (29-31). In the present study, it was revealed that CM-chitosan inhibits iNOS production and upregulates the anti-inflammatory cytokine IL-10 mRNA and protein production in LPS-induced chondrocytes. To the best of our knowledge this is a novel discovery and adds to the protective role that CM-chitosan appears to serve against OA. Similar results were reported in a previous study by Chen et al (20), in which CM-chitosan was revealed to suppress the levels of NO in IL-1 $\beta$ induced chondrocytes.

The anti-inflammatory properties of chitosan and its derivatives have been described in a number of diseases (32). Although OA is conceptualized as a non-inflammatory disease, inflammatory cytokines serve a vital role in the breakdown of proteoglycan (33). Tumor necrosis factor (TNF)- $\alpha$, IL-1 $\beta$ and IL- 6 are the main proinflammatory cytokines studied in association with OA. These cytokines have complex regulatory functions within OA $(6,24,34)$. IL-1 $\beta$ and TNF- $\alpha$ may induce MMP-mediated digestion of proteoglycan in OA $(33,35)$. IL-1 $\beta$ may induce the production of $\mathrm{NO}$ and promote apoptosis of chondrocytes (10), whereas IL-6 may reduce the expression of type II collagen and cartilage matrix proteins $(33,36)$. In the present study, the anti-inflammatory cytokine IL-10 was studied. In previous studies the downregulation of IL-10 secretion has been observed in patients with OA and a rat model of OA $(37,38)$. This downregulation of IL-10 was associated with a reduction in $\mathrm{T}$ cell immunoglobulin mucin receptor 3 expression (39). In human articular chondrocytes IL-10 may upregulate the expression of type II collagen and promote cartilage-specific extracellular matrix synthesis in response to TNF- $\alpha$ (40). In the present study, the results suggested that IL-10 activation was correlated with inhibition of iNOS production and was associated with the protective role of CM-chitosan against OA.

IL-10 interacts with its high-affinity receptor IL-10R1 and low-affinity receptor IL-10R2 (41) and leads to the activation of the JAK/STAT/SOCS signaling pathway (42). In the present study it was revealed that the protective effect of CM-chitosan was in part through the activation of the JAK/STAT/SOCS signaling pathway. Previous studies have also demonstrated that the JAK/STAT/SOCS signaling pathway was associated with iNOS production $(22,23)$.

In conclusion, the results of the present study indicated that CM-chitosan inhibited iNOS production by upregulating IL-10 and activating the JAK/STAT/SOCS signaling pathway.
However, there were some limitations to the methodology used in the present study; the experiments were performed in a cell model, an animal model should be considered for any future investigations to give a better representation of OA in humans. The expression of selected proteins was tested, but the effect of CM-chitosan on cell proliferation and extracellular matrix synthesis was not measured. Further studies into the effect of $\mathrm{CM}$-chitosan on cartilage formation are required to develop these findings.

\section{References}

1. Kidd BL: Osteoarthritis and joint pain. Pain 123: 6-9, 2006

2. Rogers MW and Wilder FV: The association of BMI and knee pain among persons with radiographic knee osteoarthritis: A cross-sectional study. BMC Musculoskelet Disord 9: 163, 2008.

3. Zhang Y and Jordan JM: Epidemiology of osteoarthritis. Clin Geriatr Med 26: 355-369, 2010

4. Le LT, Swingler TE and Clark IM: Review: The role of microRNAs in osteoarthritis and chondrogenesis. Arthritis Rheum 65: 1963-1974, 2013.

5. Wheaton AJ, Borthakur A, Shapiro EM, Regatte RR, Akella SV, Kneeland JB and Reddy R: Proteoglycan loss in human knee cartilage: Quantitation with sodium MR imaging-seasibility study. Radiology 231: 900-905, 2004.

6. Clements KM, Price JS, Chambers MG, Visco DM, Poole AR and Mason RM: Gene deletion of either interleukin-1beta, interleukin-1beta-converting enzyme, inducible nitric oxide synthase, or stromelysin 1 accelerates the development of knee osteoarthritis in mice after surgical transection of the medial collateral ligament and partial medial meniscectomy. Arthritis Rheum 48: 3452-3463, 2003.

7. Murrell GA, Jang D and Williams RJ: Nitric oxide activates metalloprotease enzymes in articular cartilage. Biochem Biophys Res Commun 206: 15-21, 1995

8. Hashimoto S, Takahashi K, Amiel D, Coutts RD and Lotz M: Chondrocyte apoptosis and nitric oxide production during experimentally induced osteoarthritis. Arthritis Rheum 41: 1266-1274, 1998.

9. Farrell AJ, Blake DR, Palmer RM and Moncada S: Increased concentrations of nitrite in synovial fluid and serum samples suggest increased nitric oxide synthesis in rheumatic diseases. Ann Rheum Dis 51: 1219-1222, 1992.

10. Vuolteenaho K, Moilanen T, Jalonen U, Lahti A, Nieminen R, van Beuningen HM, van der Kraan PM and Moilanen E: TGFbeta inhibits IL-1 -induced iNOS expression and NO production in immortalized chondrocytes. Inflamm Res 54: 420-427, 2005.

11. Gómez R, Scotece M, Conde J, Lopez V, Pino J, Lago F, Gómez-Reino JJ and Gualillo O: Nitric oxide boosts TLR-4 mediated lipocalin 2 expression in chondrocytes. J Orthop Res 31: 1046-1052, 2013.

12. Takada K, Hirose J, Yamabe S, Uehara Y and Mizuta H: Endoplasmic reticulum stress mediates nitric oxide-induced chondrocyte apoptosis. Biomed Rep 1: 315-319, 2013.

13. de Girolamo L, Kon E, Filardo G, Marmotti AG, Soler F, Peretti GM, Vannini F, Madry H and Chubinskaya S: Regenerative approaches for the treatment of early OA. Knee Surg Sports Traumatol Arthrosc 24: 1826-1835, 2016.

14. Lahiji A, Sohrabi A, Hungerford DS and Frondoza CG: Chitosan supports the expression of extracellular matrix proteins in human osteoblasts and chondrocytes. J Biomed Mater Res 51: 586-595, 2000.

15. Oprenyeszk F, Sanchez C, Dubuc JE, Maquet V, Henrist C, Compère $\mathrm{P}$ and Henrotin $\mathrm{Y}$ : Chitosan enriched three-dimensional matrix reduces inflammatory and catabolic mediators production by human chondrocytes. PLoS One 10: e0128362, 2015.

16. Xia W, Liu P, Zhang J and Chen J: Biological activities of chitosan and chitooligosaccharides. Food Hydrocolloid 25: 170-179, 2011.

17. Jung WJ and Park RD: Bioproduction of chitooligosaccharides: Present and perspectives. Mar Drugs 12: 5328-5356, 2014.

18. Kerch G: The potential of chitosan and its derivatives in prevention and treatment of age-related diseases. Mar Drugs 13: 2158-2182, 2015.

19. Croisier F and Jérôme C: Chitosan-based biomaterials for tissue engineering. Eur Polym J 49: 780-792, 2013. 
20. Chen Q, Liu SQ, Du YM, Peng $H$ and Sun LP Carboxymethyl-chitosan protects rabbit chondrocytes from interleukin-1beta-induced apoptosis. Eur J Pharmacol 541: 1-8, 2006.

21. Liu SQ, Qiu B, Chen LY, Peng H and Du YM: The effects of carboxymethylated chitosan on metalloproteinase-1, -3 and tissue inhibitor of metalloproteinase-1 gene expression in cartilage of experimental osteoarthritis. Rheumatol Int 26: 52-57, 2005.

22. Yu H, Liu Z, Zhou H, Dai W, Chen S, Shu Y and Feng J: JAK-STAT pathway modulates the roles of iNOS and COX-2 in the cytoprotection of early phase of hydrogen peroxide preconditioning against apoptosis induced by oxidative stress. Neurosci Lett 529: 166-171, 2012.

23. Dell'Albani P, Santangelo R, Torrisi L, Nicoletti VG, De Vellis J and Giuffrida Stella AM: JAK/STAT signaling pathway mediates cytokine-induced iNOS expression in primary astroglial cell cultures. J Neurosci Res 65: 417-424, 2001.

24. Séguin CA and Bernier SM: TNFalpha suppresses link protein and type II collagen expression in chondrocytes: Role of MEK1/2 and NF-kappaB signaling pathways. J Cell Physiol 197: 356-369, 2003.

25. Livak KJ and Schmittgen TD: Analysis of relative gene expression data using real-time quantitative PCR and the 2(-Delta Delta C(T)) method. Methods 25: 402-408, 2001

26. Goff WL, O'Rourke KI, Johnson WC, Lacy PA, Davis WC and Wyatt CR: The role of IL-10 in iNOS and cytokine mRNA expression during in vitro differentiation of bovine mononuclear phagocytes. J Interf Cytok Res 18: 139-149, 1998.

27. Bijlsma JW, Berenbaum F and Lafeber FP: Osteoarthritis: An update with relevance for clinical practice. Lancet 377 2115-2126, 2011

28. Zheng S, Hunter DJ, Xu J and Ding C: Monoclonal antibodies for the treatment of osteoarthritis. Expert Opin Biol Ther 16 : $1529-1540,2016$

29. Ma Z, Wang Y, Piao T and Liu J: Echinocystic acid inhibits IL-1 $\beta$-induced COX-2 and iNOS expression in human osteoarthritis chondrocytes. Inflammation 39: 543-549, 2016.

30. Cheng AW, Stabler TV, Bolognesi MP and Kraus VB: Selenomethionine inhibits IL- $1 \beta$ inducible nitric oxide synthase (iNOS) and cyclooxygenase 2 (COX2) expression in primary human chondrocytes. Osteoarthritis Cartilage 19: 118-125, 2011.

31. Ying X, Chen X, Cheng S, Shen Y, Peng L and Xu HZ: Piperine inhibits IL- $\beta$ induced expression of inflammatory mediators in human osteoarthritis chondrocyte. Int Immunopharmaco 17: 293-299, 2013.

32. Azuma K, Osaki T, Minami S and Okamoto Y: Anticancer and anti-inflammatory properties of chitin and chitosan oligosaccharides. J Funct Biomater 6: 33-49, 2015.
33. Martel-Pelletier J: Pathophysiology of osteoarthritis. Osteoarthritis Cartilage 12: (Suppl A): S31-S33, 2004.

34. Namba A, Aida Y, Suzuki N, Watanabe Y, Kawato T, Motohashi M, Maeno M, Matsumura $\mathrm{H}$ and Matsumoto $\mathrm{M}$ : Effects of IL-6 and soluble IL-6 receptor on the expression of cartilage matrix proteins in human chondrocytes. Connect Tissue Res 48: 263-270, 2007.

35. Lin PM, Chen CC and Torzilli PA: Increased stromelysin-1 (MMP-3), proteoglycan degradation (3B3- and 7D4) and collagen damage in cyclically load-injured articular cartilage. Osteoarthritis Cartilage 12: 485-496, 2004.

36. Porée B, Kypriotou M, Chadjichristos C, Beauchef G, Renard E, Legendre F, Melin M, Gueret S, Hartmann DJ, Malléin-Gerin F, et al: Interleukin-6 (IL-6) and/or soluble IL-6 receptor down-regulation of human type II collagen gene expression in articular chondrocytes requires a decrease of Sp1.Sp3 ratio and of the binding activity of both factors to the COL2A1 promoter. J Biol Chem 283: 4850-4865, 2008.

37. Imamura M, Targino RA, Hsing WT, Imamura S, Azevedo RS, Boas LS, Tozetto-Mendoza TR, Alfieri FM, Filippo TR and Battistella LR: Concentration of cytokines in patients with osteoarthritis of the knee and fibromyalgia. Clin Interv Aging 9: 939-944, 2014

38. Rojas-Ortega M, Cruz R, Vega-López MA, Cabrera-González M, Hernández-Hernández JM, Lavalle-Montalvo C and Kouri JB: Exercise modulates the expression of IL-1 $\beta$ and IL-10 in the articular cartilage of normal and osteoarthritis-induced rats. Pathol Res Pract 211: 435-443, 2015.

39. Li S, Wan J, Anderson W, Sun H, Zhang H, Peng X, Yu Z, Wang T, Yan X and Smith W: Downregulation of IL-10 secretion by Treg cells in osteoarthritis is associated with a reduction in Tim-3 expression. Biomed Pharmacothr 79: 159-165, 2016.

40. Müller RD, John T, Kohl B, Oberholzer A, Gust T, Hostmann A, Hellmuth M, Laface D, Hutchins B, Laube G, et al: IL-10 overexpression differentially affects cartilage matrix gene expression in response to TNF-alpha in human articular chondrocytes in vitro. Cytokine 44: 377-385, 2008.

41. Josephson K, Logsdon NJ and Walter MR: Crystal structure of the IL-10/IL-10R1 complex reveals a shared receptor binding site. Immunity 15: 35-46, 2001.

42. Carey AJ, Tan CK and Ulett GC: Infection-induced IL-10 and JAK-STAT: A review of the molecular circuitry controlling immune hyperactivity in response to pathogenic microbes. JAKSTAT 1: 159-167, 2012. 